\title{
The Distribution of Main Belt Asteroids with Featureless Spectra from the Sloan Digital Sky Survey Photometry
}

\author{
Anderson O. Ribeiro and Fernando Roig \\ Observatório Nacional, Rio de Janeiro, 20921-400, RJ, Brazil \\ email: anderson@on.br \\ email: froig@on.br
}

\begin{abstract}
In this work, we propose to analyse the existence of possible correlations between the taxonomic classes of asteroids showing featureless spectra -i.e. a flat continuum with no absorption bands- and their orbital properties. We compute the mean spectral slope of 14753 asteroids using the photometric data from the Sloan Digital Sky survey Moving Objects Catalog (SDSS-MOC4). Although the quality of these data is not comparable in resolution to the spectroscopic data, the amount of observations in the SDSS-MOC4 is more than 20 times larger that in the available spectral databases. This allows us to obtain a statistically significant result.
\end{abstract}

Keywords. minor planets, asteroids - surveys

\section{Introduction}

Several studies about the surface properties of Main Belt asteroids, based on spectroscopic observations, indicate that there exist correlations between the different taxonomic classes and their orbital distribution (Mothé-Diniz, Carvano \& Lazzaro, 2003; Carvano, Mothé-Diniz \& Lazzaro, 2003). However, these studies are limited by the low number of available spectroscopic data. Some 20 years ago, the known distribution of taxonomic classes was compatible with the idea that asteroids' mineralogy was correlated to the temperature gradient of the primordial nebula. Asteroids closer to the Sun showed evidences of more active heating processes, that were not detected in the more distant asteroids. But in recent years, the increasing number of observations reveal that the taxonomic classes appear uniformly distributed among the Main Belt, showing no apparent correlation to the temperature gradient of the nebula.

We propose here to analyse such correlations using the 4th release of the Moving Objects Catalog of the Sloan Digital Sky Survey (SDSS-MOC4). This catalog provides 5 band photometry of a sample of asteroids which is about 20 times larger than the spectroscopic sample.

Our analysis is limited to the so-called background asteroids, i.e. those that do not belong to any dynamical family of asteroids, and we focus on bodies with featureless spectra, i.e. those that show a flat spectrum with no evidences of absorption bands in their spectra.

\section{Methodology}

The SDSS-MOC4 provides calibrated magnitudes in the filters $u, g, r, i, z$, centred at $3540 \AA, 4770 \AA$ A, $6230 \AA, 7630 \AA$, and $9130 \AA$, respectively. From these magnitudes we compute reflectance colors and the corresponding reflectance fluxes, normalized to 1 at the $\mathrm{r}$ band. Observations with errors $>10 \%$ in any band were discarded. 
To separate the observations corresponding to featureless spectra, we apply the Principal Components Analysis (PCA). Featureless observations can be easily identified in the space os the two first principal components of the reflectance fluxes distribution.

The featureless observations were further separated into those corresponding to the members of the major dynamical families in the Main Belt and those corresponding to the background population of asteroids.

Finally, in order to characterize each featureless observation, we compute the spectral slope by fitting a straight line to the reflectance fluxes vs. wavelength.

\section{Results}

We analyze behavior of the population of background asteroids in the three regions of the Main Belt: inner, middle, and outer. Our results indicate that there are very weak correlations between the mean spectral slope and the orbital eccentricities and inclinations, both for the inner and middle Belt populations. More significant correlations are observed in the outer Belt, especially in terms of orbital inclination (Fig. 1). The wavy behavior at high inclinations shown in Fig. 1, right panel, is particularly notorious.
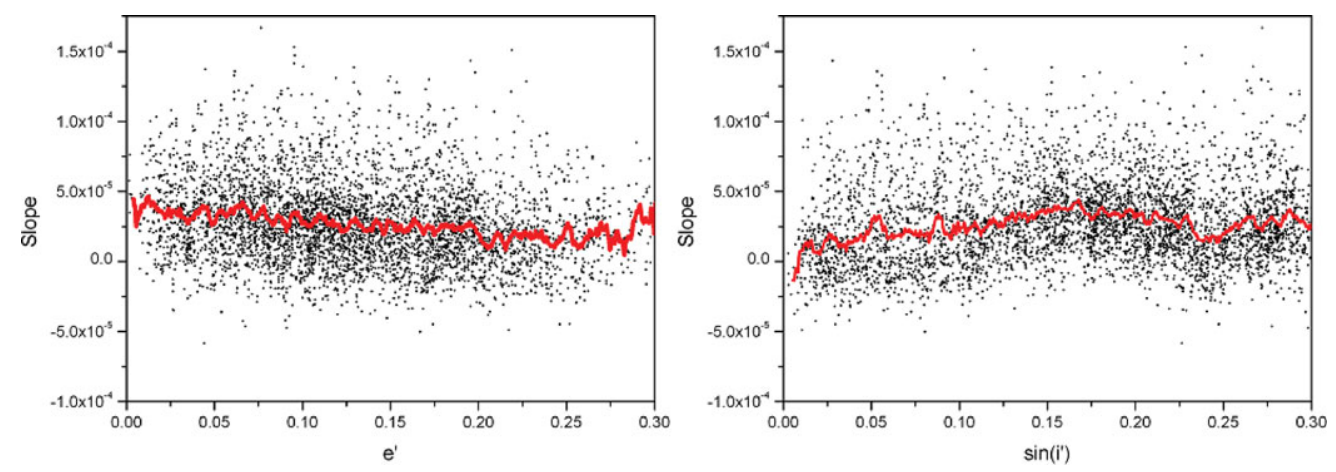

Figure 1. Correlations between mean spectral slope and proper orbital eccentricity (left), and sinus of proper orbital inclination (right), for background asteroids in the outer Main Belt with featureless spectra. The full line is the running average of the data.

A comparison with the distribution of spectral slopes of asteroids members of dynamical families in the outer Main Belt (Fig. 2), seems to indicate that the correlations observed for the background population are driven by the two major families in the region: the Themis family (high eccentricity, low inclination) and the Eos family (low eccentricity, high inclination). We propose that these two families contaminate the background population with a significant amount of fugitive asteroids, that left the presently detected dynamical families due to long term dynamical evolution, but remain in the neighborhood of their parent families.

\section{Conclusion}

We do not find any evidences of significant correlations between the spectral slopes and the proper orbital elements of background asteroids with featureless spectra in the inner and middle Belt.

In the outer Belt, the stronger correlations seem to be driven by the background contamination from the major asteroid families. We believe that this is not an artefact of the families identification process, but rather a real effect caused by the presence 

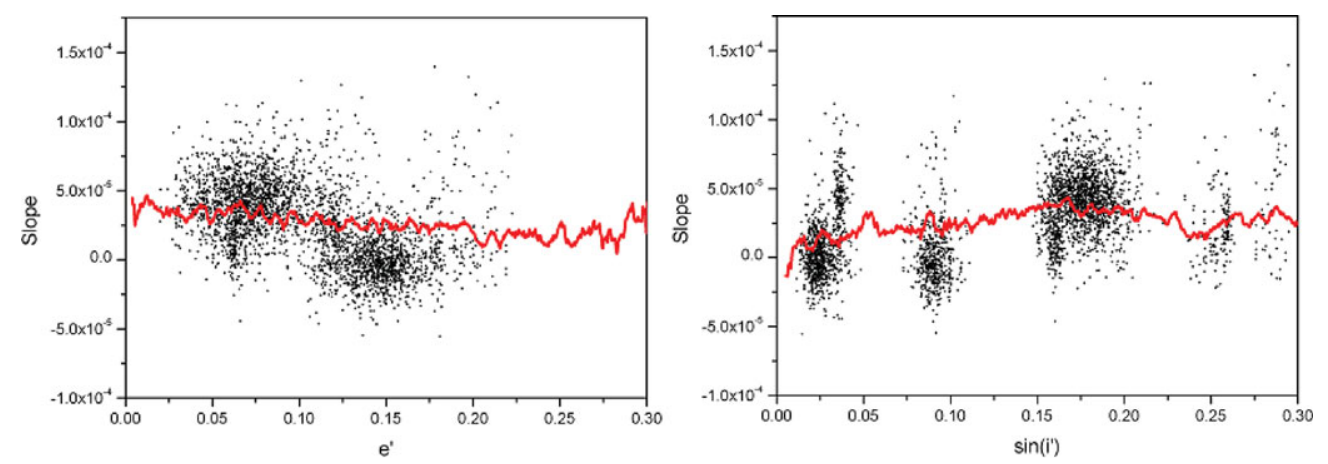

Figure 2. Correlations between mean spectral slope and proper orbital eccentricity (left), and sinus of proper orbital inclination (right), for asteroids with featureless spectra that belong to dynamical families in the outer Main Belt. The full line is the same shown in Fig. 1.

of former family members that dynamically evolved beyond the present edges of these families.

Our results tend to indicate that the actual background population of asteroids with featureless spectra are uniformly distributed in terms of eccentricities and inclinations all over the Main Belt. No correlation between spectral slopes and mean heliocentric distances has been observed either.

\section{References}

Carvano, J. M., Mothé-Diniz, T., \& Lazzaro, D. 2003, Icarus, 161, 356

Mothé-Diniz, T., Carvano, J. M., \& Lazzaro, D. 2003, Icarus, 162, 10 